\title{
Two Outbreaks of Mixed Etiology Associated with Central Venous Catheters Inserted by Phlebotomy in Critical Neonates
}

Denise V.D. de Brito ${ }^{1}$, Elias J.O. von Dolinger ${ }^{1}$, Vânia O.S. Abdallah ${ }^{1}$, Ana Lúcia C. Darini ${ }^{2}$ and Paulo P. Gontijo Filho ${ }^{1}$

${ }^{1}$ Institute of Biomedical Sciences, Federal University of Uberlândia; Uberlândia, MG; ${ }^{2}$ Faculty of Pharmaceutical Sciences of Ribeirão PretoUSP; Ribeirão Preto, SP, Brazil

\begin{abstract}
Staphylococcus aureus and coagulase-negative staphylococci are the main cause of sepsis in Neonatal Intensive Care Unit (NICU). Central venous catheters (CVCs) are an important part of critical neonates' treatment and are associated with sepsis. The aim of this study was to investigate two outbreaks caused by Staphylococcus aureus and Staphylococcus epidermidis associated with CVC inserted by phlebotomy in critical neonates. The surveillance was performed from January 2001 to December 2005 at the Brazilian NICU. The genotypic analysis of oxacillin susceptible S. aureus (OSSA) and oxacillin resistant S. epidermidis (ORSE) was performed based on pulsed-field gel electrophoresis (PFGE). Staphylococcus was the most frequent pathogen (65.8\%) with highest incidence of CoNS (59.9\%) followed by $S$. aureus $\mathbf{( 4 0 . 1 \% )}$. During the five years of surveillance, there were two outbreaks detected, occurred in January-February/02 and August/02 and confirmed by PFGE analysis. The predisposing factors for infection corresponding to both outbreaks were: age $\leq 7$ days, hospitalization $\geq 7$ days, and use of polyethylene CVC through dissection of vein (phlebotomy). This is the first relate of staphylococcal outbreaks associated with CVC inserted by phlebotomy in NICU. PFGE showed polyclonal spread of OSSA during both epidemic and endemic period, and two monoclonal outbreaks of ORSE in the same epidemic period of OSSA.

Key-Words: Outbreak, central venous catheter, neonates.
\end{abstract}

Among the agents of infectious diseases in neonates, Staphylococcus aureus and coagulase-negative staphylococci (CoNS), mainly Staphylococcus epidermidis, are emphasized as being predominantly of endemic nature [1]. Furthermore, they are the main cause of sepsis in Neonatal Intensive Care Unit (NICU) [2], and are often implied in outbreaks, with significant increase of morbidity, mortality, length of stay and cost [3].

The clonality of staphylococci has been investigated by different genotypic methods. The use of pulsed-field gel electrophoresis (PFGE) for genetic typing of staphylococci has been shown to be a suitable method in terms of its discriminatory ability [4].

Placement of central venous catheters (CVCs) is a potentially life-saving treatment for infants with poor peripheral venous access and gastrointestinal tract intolerance of adequate nutrition. However, they are associated with many complications; sepsis is the most common of them [5]. There are different kinds of CVC, according to both the forming material and the insertion method.

The aim of this study was to investigate two outbreaks caused by Staphylococcus aureus and Staphylococcus epidermidis associated with CVC inserted by phlebotomy in critical neonates.

\section{Material and Methods}

The study was performed from January 2001 to December 2005 at the NICU of a Brazilian University Hospital (Hospital

Received on 6 February 2009; revised 7 June 2009.

Address for correspondence: Dr. Denise Von Dolinger de Brito. Avenida Floriano Peixoto, $\mathrm{n}^{0}$ 5322, Bloco 09, Apt 302, Residencial Buritis, B. Umuarama. Uberlândia-MG, Brasil. Zip code: 38405-184. Phone: $\left(0^{* * 34)}\right.$ - 3232.2280 - Fax (0**34) - 3218.2333. E-mail: denisebrito@terra.com.br.

The Brazilian Journal of Infectious Diseases 2009;13(3):177-182. (C) 2009 by The Brazilian Journal of Infectious Diseases and Contexto Publishing. All rights reserved. de Clínicas da Universidade Federal de Uberlândia), an 11bed level III neonatal reference center.

The epidemiologic surveillance was performed according to the National Nosocomial Infection Surveillance System (NNISS) with daily visits to the unit to search nosocomial infections and the risk factors associated. In the hospital laboratory we obtained staphylococci cultures isolated from infections in neonates hospitalized in the period of 2001-2005. Both outbreaks were defined by statistical analysis ( $\mathrm{p} \leq 0.05)$, when compared with the endemic period. Case-control study was used where cases included 26 neonates with clinic and microbiologic diagnostic of staphylococcal infection, and control neonates hospitalized in the same period without diagnostic of infection.

In the period of study, infants admitted to the unit were included into this surveillance. The diagnosis of sepsis caused by staphylococci required clinical signs of sepsis in a neonate older than 72 hours of age and a positive monomicrobial blood culture as well as elevated C-reactive protein (CRP) $>10 \mathrm{mg} / \mathrm{L}$ within 2 days of blood culture.

The criteria for diagnosis of specific infections like conjunctivitis, otitis and rinitis used clinical diagnosis first and then microbiological diagnosis. Radiological and clinical criteria were used to identify pneumonia.

Parents of neonates in the NICU were given a letter describing the study. This study was approved by the Research Ethics Committee of Uberlândia University Hospital.

\section{Microbiological Studies}

Blood cultures of staphylococci were obtained and neonates were enrolled into the study as cases of staphylococcal sepsis. Blood cultures were processed in the Laboratory of Microbiology, Uberlândia University Hospital. Blood culture bottles were plated on trypticase soy agar with 
$5 \%$ sheep blood, and the results were incubated for 24 hours at $35^{\circ} \mathrm{C}$ and were visually examined to identify presumptive staphylococci on the basis of colonial morphology and catalase production. Staphylococcus isolates were identified as $S$. aureus by the manitol fermentation, DNase and coagulase activity (free and conjugate).

All CoNS blood culture isolates were identified in species by the detection of enzymes (coagulase, catalase, phosphatase, ornitine, urease and pyrrolidinyl arylamidase), hemolytic activity, production of acid from manitol, manose and trealose, polymixine and desferrioxamine resistance and confirmed by API STAPH ID32 system (bioMérieux Vitek, Australia) following the manufacturer's instructions.

The antimicrobial sensitivity of the Staphylococcus isolates in the epidemic period was determined by the disk diffusion method using the following disks (OXOID LTD., Basingstoke, Hampshire, England): ampicillin, oxacillin, gentamicin, ciprofloxacin, sulfazotrin, clindamicin, tetracycline, chloramphenicol, rifampicin, vancomycin, imipenem and ampicillin/sulbactam and by the dilution method using the oxacillin (OXOID LTD., Basingstoke, Hampshire, England) at concentrations from $0.25 \mu \mathrm{g} / \mathrm{mL}$ to $1024 \mu \mathrm{g} / \mathrm{mL}$ which were interpreted according to the Clinical and Laboratory Standards Institute [6].

\section{PFGE}

The genotypic analysis of oxacillin susceptible $S$. aureus (OSSA) and oxacillin resistant S. epidermidis (ORSE) was performed based on pulsed-field gel electrophoresis (PFGE). The fragments of DNA were generated by digestion with restriction enzyme SmaI using the Gene Navigator apparatus (Pharmacia Biotech). The criteria to definition of genetic profiles were defined by Tenover et al., 1995 [7].

Polymerase Chain Reaction (PCR)-Based Detection of the mecA Gene of CoNS

The polymerase chain reaction (PCR) to detection of the mec $A$ gene of CoNS was according to Nunes et al., 2005 [4].

\section{Statistical Analysis}

Analyses of occurrence outbreaks were performed by application of endemic curve with Poisson's distribution [8].

Analyses of risk factors for infection were performed using the $\chi^{2}$ - test and Fisher's exact test. The risk factors that showed significance $(\mathrm{p} \leq 0.05)$ in the univariated analysis were revaluated using a logistic regression model (multivariate analysis) through the statistical package SPSS (Version 10).

\section{Results}

The average of admissions per month was 32.6. The unit has five nurses, four sinks with five sinks at the entrance with paper towels, automated faucet and dispensers of $2.0 \%$ chlorhexedine. The time of average permanence in the unit was 23 days with 3.508 ventilatordays and 6.532 CVC-days.
From January 2001 to December 2005, 610 (31.2\%) hospital acquired infections were detected among 1957 neonates from which 350 (17.9\%) infections were laboratory confirmed. Sepsis was the most frequent infectious syndrome (69.2\%) and Staphylococcus was the most frequent pathogen (65.8\%) with highest incidence of CoNS (59.9\%) followed by S. aureus $(40.1 \%)$. All the isolates of the outbreaks were from bloodstream and $81.8 \%$ were CVC related.

The majority (89,9\%) of the CoNS isolates were identified as $S$. epidermidis, most of which were oxacillin- and multiresistant (73.4\%). The detection of oxacillin resistance as determined by the disk-diffusion method correlated with the presence of the mecA gene detected by PCR. The oxacillin minimal inhibitory concentration (MIC) ${ }_{90}$ concerning isolates was $256 \mu \mathrm{g} / \mathrm{mL}$. While the ORSE isolates showed multiresistance, $S$. aureus isolates showed high resistance

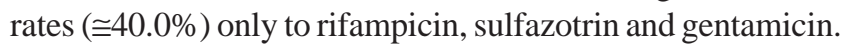
The oxacillin minimal inhibitory concentration (MIC) ${ }_{90}$ to $S$. aureus isolates was $0.25 \mu \mathrm{g} / \mathrm{mL}$.

Two outbreaks of mixed etiology by OSSA and ORSE were detected, through surveillance performed by microbiology laboratory, corresponding respectively to the periods of January-February/02 and August/02, defined by endemic curve.

The epidemiological investigation of two outbreaks was performed using case-control study (Table 1). The predisposing factors for infection corresponding to both outbreaks were: age $\leq 7$ days [p,0.0001, OR 0.04 (0.01-0.17)], hospitalization $\geq 7$ days [ $\mathrm{p}<0.0001$, OR 11.40(3.21-43.12)], and use of polyethylene CVC through dissection of vein (phlebotomy) [p<0.0001, OR 13.57 (3.56-56.47)] (Table 2). There was not independent risk factor by multivariate analysis. Of 22 epidemic cases of staphylococci, 18 (81,8\%) were using CVC inserted by phlebotomy with 518 catheters days. The other four cases were using peripherally insertion central catheter (PICC). There was not the use of another invasive procedure. The first outbreak (Jan/Feb 02) was solved out in March/02 by continuous utilization of PICC, but because of the suspension of this use in August/02, the occurrence of the second outbreak by staphylococci was seen. Containment of the outbreaks was achieved by strict hygienic measures like cohort nursing of the infected infants and hand washing before and after contact with neonates, beyond the use of CVC type less aggressive (PICC) and training of staff nursing in the moment of CVC insertion.

Sma I digestion of bacterial DNA to PFGE was performed on 18 OSSA isolates, including six of endemic and 12 of epidemic period and the predominance of a polyclonal pattern was observed (Figure 1). The PFGE analysis of SmaI digests of genomic DNA of the 49 S. epidermidis isolates, resulted in detection of two outbreaks occurred in January-February/2002 and August/02 with seven strains in each, of monoclonal nature (Figure 2 dendrogram). Additionally, small clusters were detected into the epidemic period related to the $S$. aureus (isolates numbers 20, 17 and isolates 23, 24, 30) and $S$. epidermidis (isolates numbers 41, 40, 39 and isolates 57, 56). 
Table 1. Staphylococci infection of endemic and epidemic nature in the Neonatal Intensive Care Unit (NICU) of Uberlândia Federal University Hospital during the period from 2001 to 2002.

\begin{tabular}{|c|c|c|c|c|c|c|}
\hline \multirow[t]{2}{*}{ Infection } & \multicolumn{3}{|c|}{ S. aureus } & \multicolumn{3}{|c|}{ CoNS } \\
\hline & $\begin{array}{c}\text { Epidemic } \\
\mathrm{N}=12(\%)\end{array}$ & $\begin{array}{c}\text { Endemic } \\
\text { N=18 (\%) }\end{array}$ & $\begin{array}{c}\text { Total } \\
\mathrm{N}=30(\%)\end{array}$ & $\begin{array}{c}\text { Epidemic } \\
\mathrm{N}=14(\%)\end{array}$ & $\begin{array}{c}\text { Endemic } \\
\mathrm{N}=33(\%)\end{array}$ & $\begin{array}{c}\text { Total } \\
\mathrm{N}=47(\%)\end{array}$ \\
\hline Sepsis & $8(66.7)$ & 12 (66.7) & 20 (66.7) & 13 (92.9) & 22 (66.7) & 35 (74.5) \\
\hline Conjunctivitis & $3(25.0)$ & 4 (22.3) & $7(23.3)$ & $1(7.1)$ & 10 (30.3) & 11 (23.4) \\
\hline Pneumonia & - & $1(5.5)$ & $1(3.3)$ & - & - & - \\
\hline Otitis & - & & - & - & $1(3.0)$ & $1(2.1)$ \\
\hline Rinitis & $1(8.3)$ & $1(5.5)$ & $2(6.7)$ & - & - & - \\
\hline
\end{tabular}

CoNS = coagulase-negative Staphylococci.

Table 2. Risk factors for acquisition of OSSA and ORSE infections in neonates hospitalized in the Neonatal Intensive Care Unit (NICU) of Uberlândia Federal University Hospital during the period of January-February/02 and August-September/02.

\begin{tabular}{|c|c|c|c|c|}
\hline Risk factor & $\begin{array}{c}\text { Cases } \\
\mathrm{N}=26(\%)\end{array}$ & $\begin{array}{c}\text { Controls } \\
\mathrm{N}=52(\%)\end{array}$ & $\mathrm{OR}\left(\mathbf{C I}_{95 \%}\right)$ & $\mathbf{P}$ \\
\hline \multicolumn{5}{|l|}{ Weight } \\
\hline$>1500 \mathrm{~g}$ & $9(34.6)$ & 30 (57.7) & $0.39(0.13-1.14)$ & 0.05 \\
\hline$\leq 1500 \mathrm{~g}$ & 17 (65.4) & 22 (42.3) & & \\
\hline \multicolumn{5}{|l|}{ Sex } \\
\hline Feminine & $11(42.3)$ & $28(53.8)$ & $0.63(0.22-1.80)$ & 0.33 \\
\hline Masculine & 15 (57.7) & $24(46.2)$ & & \\
\hline \multicolumn{5}{|l|}{ Age } \\
\hline$>7$ days & 5 (19.2) & $44(84.6)$ & $0.04(0.01-0.17)$ & $<0.0001^{*}$ \\
\hline$\leq 7$ days & $21(80.8)$ & 8 (15.4) & & \\
\hline \multicolumn{5}{|l|}{ Gestation (weeks) } \\
\hline 26-29 & $6(23.1)$ & $13(25.0)$ & $0.9(0.26-3.07)$ & 0.85 \\
\hline $30-33$ & $6(23.1)$ & 22 (42.3) & $0.41(0.12-1.32)$ & 0.09 \\
\hline $34-36$ & 14 (53.8) & 17 (32.7) & $2.40(0.82-7.07)$ & 0.07 \\
\hline \multicolumn{5}{|c|}{ Length of hospitalization } \\
\hline$\geq 7$ days & $21(80.8)$ & $14(26.9)$ & $11.4(3.21-43.12)$ & $<0.0001^{*}$ \\
\hline \multicolumn{5}{|l|}{ Apgar in the 5 , } \\
\hline $0-4$ & $5(19.2)$ & $13(25.0)$ & $0.71(0.19-2.57)$ & 0.56 \\
\hline $5-7$ & $9(34.6)$ & $19(36.5)$ & $0.92(0.31-2.74)$ & 0.86 \\
\hline $8-10$ & 12 (46.1) & $20(38.5)$ & $1.37(0.48-3.96)$ & 0.51 \\
\hline Incubator & $18(69.2)$ & 33 (63.5) & $1.30(0.43-4.0)$ & 0.61 \\
\hline \multicolumn{5}{|l|}{ Antibiotic use } \\
\hline Ampicillin & 25 (96.2) & 40 (76.9) & 7.50 (0.91-163.7) & 0.03 \\
\hline Amicacin & 25 (96.2) & 40 (76.9) & 7.50 (0.91-163.7) & 0.03 \\
\hline$>2$ antibiotics & $18(69.2)$ & $36(69.2)$ & $1.0(0.32-3.14)$ & 1.0 \\
\hline \multicolumn{5}{|l|}{ Use of } \\
\hline CVC & $22(84.6)$ & 15 (28.8) & 13.57 (3.56-56.47) & $<0.0001^{*}$ \\
\hline PVC & $24(92.3)$ & 40 (76.9) & $3.60(0.67-25.55)$ & 0.09 \\
\hline
\end{tabular}

$* \mathrm{p}=0.05$; OSSA $=$ oxacillin susceptible Staphylococcus aureus; ORSE = oxacillin resistant Staphylococcus epidermidis; OR= odds ratio; $\mathrm{CI}_{95 \%}=95 \%$ confidence intervals; $\mathrm{CVC}=$ central vascular catheter; $\mathrm{PVC}=$ peripheral venous catheter.

\section{Discussion}

The infections in premature neonates are most frequent and habitually most serious, associated with high mortality [9]. The prolonged period of hospitalization of very low birth weight babies ( $\mathrm{w}<1000 \mathrm{~g}$ ), in addition to both immunological immaturity and demand of intravascular catheter are the responsible set for a major susceptibility to microorganism and the occurrence of outbreaks [10].
Two outbreaks associated to bloodstream infections were detected within the period of two years (20012002) at the Neonate Intensive Care Unit (NICU) of Uberlândia Federal University Hospital, from which, two were of mixed etiology of OSSA and ORSE, defined by endemic curve. The incidence rates of $S$. aureus and CoNS were $40.1 \%$ and $59.9 \%$, respectively, in the investigated period. 
Figure 1. PFGE profiles of SmaI-digested genomic DNA of 18 Staphylococcus aureus strains. (a) Lanes M represent the concatamer of DNA lambda; lanes 7 to 19 contain the digested DNA of endemic period and lanes 20 to 31 contain the digested DNA of epidemic period. (b) Dendrogram resulting from the computer-assisted analysis of the PFGE profiles.

A

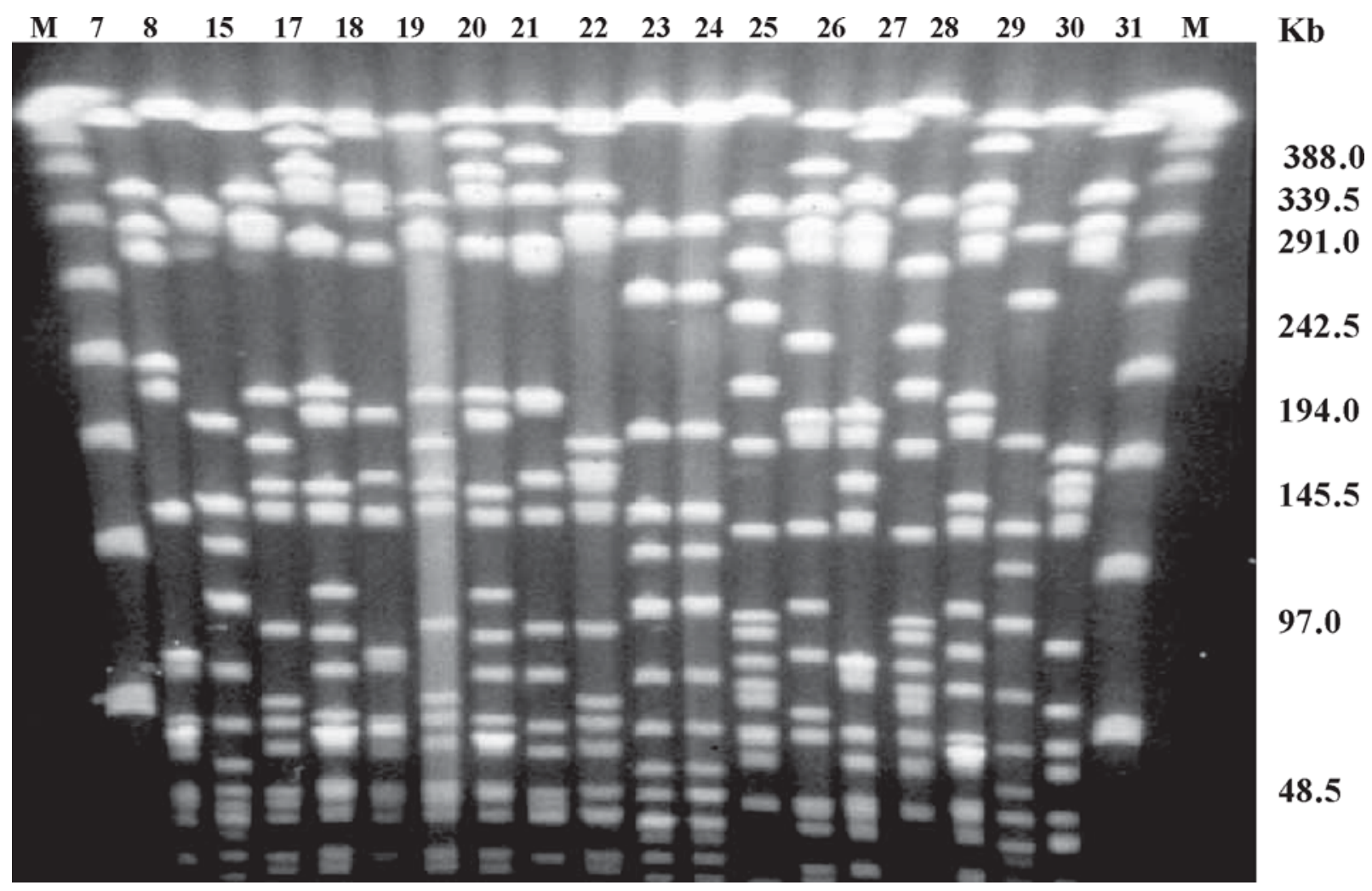

B

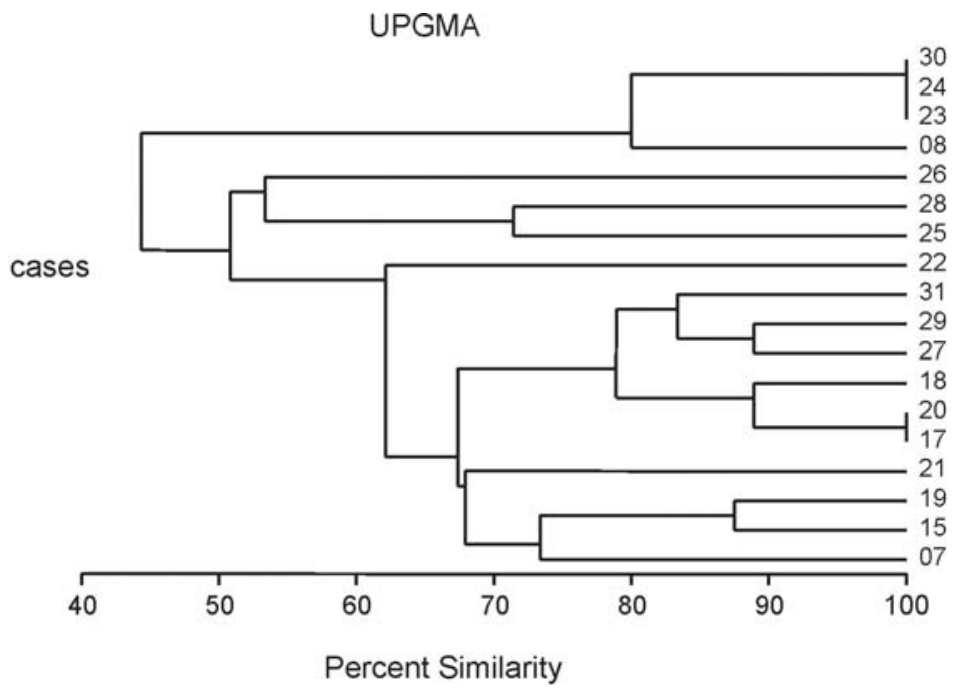


Figure 2. Percent similarity resulting from the computer-assisted analysis of the PFGE profiles of SmaI-digested genomic DNA of Staphylococcus epidermidis strains. Isolates number 7 to 12 and 15 were from first outbreak, isolates number 45 to 51 were from the second outbreak. The other isolates listed were from the endemic period.

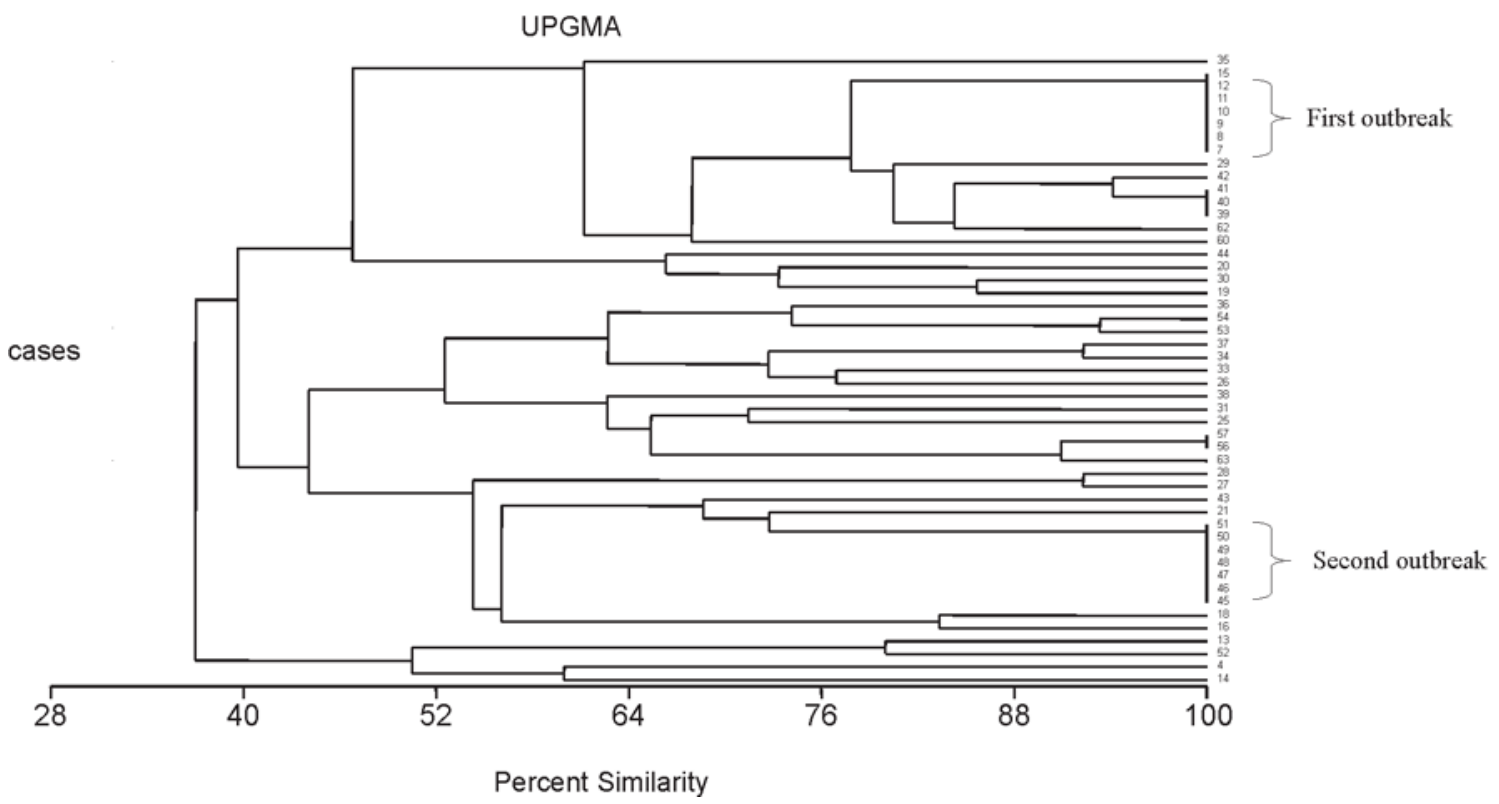

Bloodstream infection is one of the most common serious bacterial infections in hospitalized infants [11] and $S$. epidermidis is one of the major agents especially in premature neonates [12], but usually without relationship with outbreaks [1]. On the other hand, S. aureus also represents not only one of the main agents of infection in these units but in many cases it is also associated to epidemics [13] S. epidermidis is considered the main cause of late sepsis in NICUs, connected with neonates of low weight $[14,15]$. In this series, sepsis (69.2\%) was the most frequent episode in neonates.

According to reports of the country [16] a predominance of oxacillin resistant Staphylococcus aureus (ORSA) was verified in the cases of sepsis by $S$. aureus in neonates hospitalized on the contrary of those seen in our investigation, in which this phenotype was not detected. Additionally, infection by ORSA is more commonly associated with adult patients, particularly in those with the following risk reports: prolonged hospital stay, antimicrobial use, invasive procedures, surgeries and patients submitted to hemodialysis or peritoneal dialysis [17].

Consistent with previous studies from NICUs, we found a high overall level of antibiotic resistance among the $S$. epidermidis isolates in this study related to two outbreaks, representing $73.4 \%$ of infection episodes which is indicative of increased transmission rates of them and simultaneously high antimicrobial usage, mainly oxacillin, cephalosporins and/ or aminoglycosides (56.5\%) prevalence rate. There may be different explanations for this observation. Sepsis neonates were more premature, received more invasive procedures like $\mathrm{CVC}$ and were more exposed to prophylatic antibiotic, which in turn may lead to selection of resistant microbes [10].
Surveillance of outbreaks and identification of risk factors are essential if effective preventive strategies are to be developed and evaluated [10]. Our results showed the following risk factors associated to cases referring to two outbreaks: central vascular catheter (CVC) inserted using the phlebotomy technique, hospitalization $\geq 7$ days and age $\leq 7$ days. Nevertheless, none of them were independent risk factors by multivariate analysis. This is the first relate of staphylococcal outbreaks associated with CVC inserted by phlebotomy in NICU emphasizing that the dissection of vein increases the risk of infection. The rate of bacteraemia CVC-related/1000 CVC-days was 54.5 in the five years of surveillance. The outbreaks were controlled in two opportunities by re-establishment of PICC use due to constant need of central catheter. The insertion of catheter by dissection (phlebotomy) increases in at least six times the risk of infection when compared with peripherally inserted central catheter (PICC) [18]. Additionaly, strict hand washing and cohort nursing of the infected infants helped put an end to the outbreaks.

Analysis of chromosomal DNA profiles by PFGE has been shown to be a useful tool for epidemiological typing and investigation of the source, transmission and spread of nosocomial infections caused by staphylococci [4]. Although the observed one in literature where $S$. aureus outbreaks in UTINs are generally monoclonal [19] and S. epidermidis outbreaks are polyclonal [20], in this study PFGE showed polyclonal spread of OSSA during both epidemic and endemic period, and two monoclonal outbreaks of ORSE in the same epidemic period of OSSA. 


\section{Acknowledgements}

We are grateful to Joseane Cristina Ferreira for her technical assistance during the PFGE assay.

\section{References}

1. Makhoul I.R., Smolkin T., Sujov P., et al. PCR- based diagnosis of neonatal staphylococcal bacteremias. J Clin Microbiol 2005;43:4823-5.

2. Spanu T., Sanguinetti M., D’Inzeo T., et al. Identification of methicillin-resistant isolates of Staphylococcus aureus and coagulase negative Staphylococcus responsible for bloodstream infections with Phoenix ${ }^{\mathrm{TM}}$ system. Diagn Microbiol Infect Dis 2004;48:221-7.

3. Lally R.T., Lanz E., Schrock C.G., Minnesota R. Rapid control of an outbreak of Staphylococcus aureus on a Neonatal Intensive Care department using standard infection control practices and nasal mupirocin. Am J Infect Control 2004;32:44-7.

4. Nunes A.P.F., Teixeira L.M., Bastos C.C.R., et al. Genomic characterization of oxacillin-resistant Staphylococcus epidermidis and Staphylococcus haemolyticus isolated from Brazilian medical centres. J Hosp Infect 2005;59:19-26.

5. Klein M.D., Rood K., Graham P. Central venous catheter sepsis in surgical newborns. Pediatr Surg Int 2003;19:529-32.

6. Clinical and Laboratory Standards Institute. Methods for dilution antimicrobial susceptibility tests for bacteria that grow aerobically. $5^{\text {th }}$ ed., v.20, Approved Standard M7-A5. Wayne, Pennsylvania, 2005.

7. Tenover F.C., Arbeit R., Goering R.V., et al. Interpreting chromosomal DNA restriction patterns produced by pulsed field gel electrophoresis criteria for bacterial strain typing. J Clin Microbiol 1995;33:2233-9.

8. Sellick J.A. The use of statistical process control charts in hospital epidemiology. Infect Control Hosp Epidemiol 1993;14:649-56.

9. Harris J.S. Pediatrics nosocomial infections: children are not little adults. Infect Control Hosp Epidemiol 1997;18:739-42.

10. Pawa A.K., Ramji S., Prakash K., Thirupuram S. Neonatal nosocomial infection: profile and risk factors. Ind Pediatr 1997;34:297-302.
11. Graham P.L., Morel A.S., Zhou J., Wu F. et al. Epidemiology of methicillin-susceptible Staphylococcus aureus in the Neonatal Intensive Care Unit. Infect Control Hosp Epidemiol 2002;23:677-82.

12. Villari P., Sarnataro C., Iacuzio L. Molecular epidemiology of Staphylococcus epidermidis in a Neonatal Intensive Care Unit over a three year period. J Clin Microbiol 2000;38:1740-6.

13. Corbella X., Dominguez M.A., Pujol M. et al. Staphylococcus aureus nasal carriage as a marker for subsequent staphylococcal infections in intensive care unit patients. Eur J Clin Microbiol Infect Dis 1997; $16: 351-7$.

14. Raimundo O., Heussler H., Bruhn J.B. et al. Molecular epidemiology of coagulase-negative staphylococcal bacteraemia in a newborn intensive care unit. J Hosp Infect 2002;51:33-42.

15. Andersen B.M., Lindemann R., Bergh K. et al. Spread of methicillinresistant Staphylococcus aureus in a neonatal intensive unit associated with understaffing, overcrowding and mixing of patients. J Hosp Infect 2002;50:18-24.

16. Loureiro M.M., Moraes B.A., Quadra M.R.R. et al. Study of multidrug resistant microorganisms isolated from blood cultures of hospitalized newborns in Rio de Janeiro city, Brazil. Braz J Microbiol 2002;33:73-8.

17. Talon D., Woronoff-Lemsi M.C., Limat S. et al. The impact of resistance to methicillin in Staphylococcus aureus bacteremia on mortality. Europ J Intern Med 2002;13:31-6.

18. Vergunta R.K., Loethen P., Wallace L.J. et al. Differences in the outcome of surgically placed long-term central venous catheters in neonates: neck vs groin placement. J Pediatr Surg 2005;40:47-51.

19. Bertin M.L., Vinski J., Schmitt S. et al. Outbreak of methicillinresistant Staphylococcus aureus colonization and infection in a neonatal intensive care unit epidemiologically linked to a healthcare worker with chronic otitis. Infect Control Hosp Epidemiol 2006;27:581-5.

20. Björkqvist M., Söderquist B.O., Törnqvist E.V.A. et al. Phenotypic and genotypic characterization of blood isolates of coagulase-negative staphylococci in the newborn. APMIS 2002;110:332-9 